\title{
Call for Papers
}

\section{"Person-Oriented Research Methods"}

\section{A Topical Issue of the Zeitschrift für Psychologie / Journal of Psychology}

Person-oriented research is based on the fact that statements at the aggregate level rarely apply at the level of smaller numbers of cases or individuals. The first tenet of person-oriented research proposes that structure and development of behavior are, at least in part, specific to the individual. While aggregate-level statements may be correct, they cover only that portion of the overall variability that is shared by the aggregate. Larger, and more important portions of variability may remain unexplained. Recent research has already created many examples that show that individual characteristics may not be captured at all when results that were created at the aggregate level are used for description at the individual level. We are inviting contributions to a topical issue of the Zeitschrift für Psychologie / Journal of Psychology on "Approaches to data analysis: Looking at variables and persons." With this issue, we pursue three aims:

1. To provide an overview of theoretical and methodological developments of person-oriented research.

2. To present recent and new developments in the domain of statistical analysis of data from a person-oriented perspective.

3. To present empirical sample cases in which methodological implications of the relationship between aggregatelevel and person-oriented research are defined and illustrated.

Original contributions to any of these aims are invited. There is no constraint on the domain of psychology or statistical method of analysis. There is a three-stage submission process for this issue. Initially, authors are requested to submit a 200-word abstract of their proposed paper, which will be reviewed within 2 weeks after the first deadline. Authors who are requested to submit full papers should then do so within the second deadline. All papers will undergo full peer review and receive a decision within 4 weeks of the second deadline. Once a manuscript has been accepted, the authors have a third deadline by which they must send the final version of their article. Inquiries and abstracts can be addressed to Alexander von Eye at voneye@msu.edu.

Deadline for submission of abstracts is July 15, 2009. Deadline for submission of full papers is Nov 15, 2009. Deadline for final versions of selected articles is February 15, 2010

The journal seeks to maintain a short turnaround time, with the final version of the accepted papers being due by February 15,2010 . The topical issue will be published as Issue 3 (2010).

The Zeitschrift für Psychologie / Journal of Psychology was founded in 1890 and is the second oldest psychology journal in the world. One of the founding editors was Herrmann Ebbinghaus. Since 2007, it has been published in English and is devoted to publishing topical issues that provide state-of-the-art reviews of current research in psychology.

\section{Guidelines for article preparation:}

- Only English-language submissions can be considered.

- Contributions must be original (not published previously or currently under review for publication elsewhere).

- Regular articles should not exceed 45,000 characters and spaces in length, including references, figures, and tables (allowances for figures and tables should be deducted on the basis of size - approximately 1,250 characters for a quarter-page figure/table).

- Other submission formats (research summaries, opinion pieces, etc.) may be considered; please contact the Editors for details.

- Reference citations in the text and in the reference list should be in accordance with the principles set out in the Publication Manual of the American Psychological Association (5th ed.) - see also any recent issue of the journal.

For detailed author guidelines, please see the journal's website at www.hhpub.com/journals/zfp/ 